\title{
The application status and prospects of vibro ripper
}

\author{
Wang Kaile ${ }^{1,}$ *, Yang Guoping ${ }^{1}$, Hu Kaijun ${ }^{1}$, Wang Kai ${ }^{1}$, Cao Guojun ${ }^{2}$ \\ ${ }^{1}$ Shanghai University of Engineering Science, Shanghai, 201620, China \\ ${ }^{2}$ Shanghai Shing Machinery Co. Ltd, Shanghai, 201804, China
}

\section{Email address:}

kaile5211@126.com (W. Kaile), ygpljyl@sues.edu.cn (Y. Guoping), 1051627360@qq.com (H. Kaijun), 1158698182@qq.com (W. Kai), jxtracy8309@sina.com (C. Guojun)

\section{To cite this article:}

Wang Kaile, Yang Guoping, Hu Kaijun, Wang Kai, Cao Guojun. The Application Status and Prospects of Vibro Ripper. International Journal of Energy and Power Engineering. Vol. 3, No. 3, 2014, pp. 158-161. doi: 10.11648/j.ijepe.20140303.18

\begin{abstract}
Vibro ripper is a new pattern of breaker hammer, The hydraulic pump is the power source, Hydraulic oil as the working medium. By the hydraulic energy into mechanical energy of actuator mechanism, It works by the impact force, Vibro ripper combined with the use of excavators. The crushing effect is amazing. This paper introduced the domestic of research and development status of the vibro ripper home and abroad. The key technology of the vibro ripper were summarized and analyzed. Put forward the development trend base on these aspect.
\end{abstract}

Keywords: Vibro Ripper, Actuator Mechanism, Piston Breaker, Efficiency

\section{Introduction}

As a new type of hydraulic engineering equipment hydraulic breaker hammer, Crusher is widely used in mine rock, project construction, especially the transformation of the old city, broken of concrete member ${ }^{[1]}$. Due to the diversity of its carrier equipment, play the effective in labor productivity and job flexibility. With the control of blasting quarrying method worldwide, the vibro ripper has got in the teeth of the storm and got attention unprecedented.

\section{The Research Survey of the Vibro Ripper}

\subsection{Situation in Abroad}

In 1999, ThyssenKrupp company put forward a theory base on alternating stress wave. The efficiency of new breaker type is up to 4 times higher than traditional piston breaker. Prototype testing found that the effect of soft rock is pretty well, but when comes to hard rock, the result is unsatisfactory. The companies are not satisfied with the results. And open the drawing to peer. Spain's Tabe Company and Xcentric company, South Korea Cable Company, integrating this technology and continue to develop. Gradually become several different technical routes.
Spain's Tabe Company basically followed the layout of the structure of German ThyssenKrupp. But the circumferential constraints conflict excitation box straight reciprocating motion trajectory and connecting rod. The configuration lead to a part of force is not hit the stone. Blow in the bearing and the connecting rod. Spain's Xcentric company, they in one's integrity in the technical basis of German drawings, in order to overcome the stress wave damage to ripper fatigue, Only thick steel plate overall, So the product is heavy and the efficiency of general.

\subsection{The Domestic Situation}

The research work Chinese vibro ripper begins around 2000,integrating the layout of the structure and experimental data of German ThyssenKrupp. That is to say that we have kept pace with the world. The rapid development of vibro ripper and the technological tend to improvement. For example, Shanghai Shing Machinery Co. Ltd, Shanghai FenYi Machinery Co. Ltd, Ganzhou LiJian Machinery Co. Ltd, and so on. These companies have been investing research and development .

The Shing Machinery is based on original drawings of the project, and using the improved scheme. The form of the three eccentric vertical balance of inertial energy storage and 
impact piston is used by FenYi Machinery Co., Ltd. That is to say that China is one of the best vibro ripper makers.

\section{The Basic Structure and Working Principle of the Vibro Ripper}

\subsection{The Structural of the Vibro Ripper}

The vibro ripper is consists of a vibration exciter, drag link mechanism, knife board, aseismic airbag, bucker teeth, hydraulic motor and bedplate. The vibration exciter is arranged on the crushing mechanism and the static connection. The breaking plant and the drag link mechanism is the dynamic connection structure. The damping mechanism is arranged on the upper surface of the crushing mechanism mounting table. As shown in "Fig. 1". The crushing mechanism is the key components ${ }^{[2]}$.

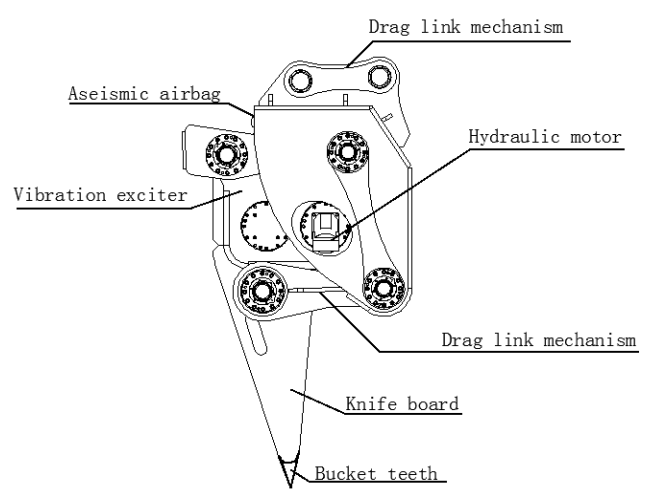

Figure 1. The constitution of the vibro ripper

\subsection{The Working Principle of the Vibro Ripper}

Vibro ripper has changed the traditional form of the working principle of reciprocating piston, the crushing force is derived from the high speed vibration of exciter.As one of the crushing plant, the vibro ripper is a physical device which is controlled by mechanical hydraulic drive system and digital signal ${ }^{[3]}$. The hydraulic motor is supply for energy which drives the eccentric block. The excavator offers the energy to hydraulic motor, so the final energy is provided by the excavator hydraulic system. Two eccentric wheels through a pair of meshing gears to rotate synchronously in opposite directions. A pair of eccentric wheel produces centrifugal force at the same time. When the switch to horizontal position, centrifugal force is counteracted in horizontal direction, when the eccentric rotating in vertical direction, the centrifugal force superimposed in the vertical direction. As shown in "Fig. 2 and Fig. 3". The impact energy is proportional to the speed of the hydraulic servomotor. Fsin $\omega t$ is the interference force changes periodically, the force which make the shaft vibration pressure is called excitation force. The box body vibration exciter to transmit the vibration to the bucket teeth. In this way, the crushing force could let the object from the internal crack. The exciting force calculation formula is as follow;

$$
\mathrm{F}=2 \mathrm{me} \omega^{2} \sin \omega \mathrm{t}
$$

In the formula, $\mathrm{m}$ is the quality of the eccentric block, $\mathrm{e}$ is the eccentric distance of eccentric block, $\boldsymbol{\omega}$ is the rotate speed of eccentric block. When a certain pressure, the greater the follow is, the greater crushing forces the gain.

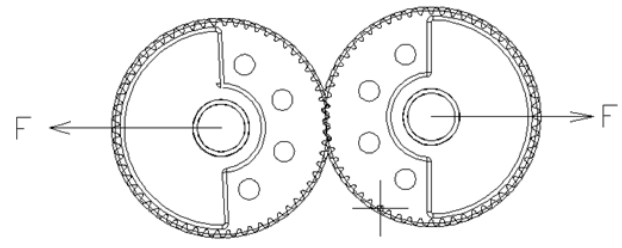

Figure 2. The exciting force in horizontal

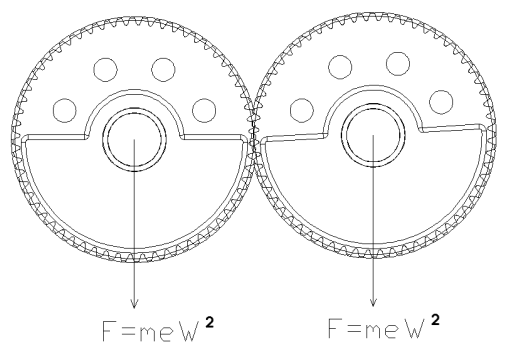

Figure 3. The exciting force in vertical

\section{The Advantage and Application of Vibro Ripper}

\subsection{The Common Rock Mohs' Hardness}

Vibro ripper is widely used in mine rock, project construction, especially the transformation of the old city, broken of concrete member. The mining and road construction need the broken rock as a rule; these rocks are common broken objects in the table below, the mohs' hardness as shown in Table [1].

Table 1. Mohs' hardness reference table

\begin{tabular}{ll}
\hline Mohs' hardness & standard mineral \\
\hline 1-3 hardness level & Talc, Gypsum \\
3-4 hardness level & Red sand rock, Fluorite \\
5-6 hardness level & Apatite, Syenite \\
7-8 hardness level & Quartz, Topaz \\
9-10 hardness level & Marble, Adamas \\
\hline
\end{tabular}

As can be seen form Table 1, the piston breaker commonly working mainly in hardness and concentrated in the 3-8 hardness level of rock, these rocks completely could be broken in the rang of the vibro ripper, when works on the rocks which the hardness below 5 level(the tensile below 
$100 \mathrm{Mpa}$ ), it can fracture quickly. With the hardness increasing, the efficiency of vibro ripper slowing down. The depth is about $50-70 \mathrm{~cm}$ is the best each broken.

\subsection{Rating of Merit}

The piston breaker originated during World War II, it has developed 50-60 years. Compare with the traditional piston breaker, the vibro ripper enhances 3-5 times higher output, reduces synthesize fuel consumption and reduces unit output emission load and minimum operating noise. We get the efficiency of the virbo ripper compares with piston hammer and blasting by Shanghai Shing Machinery Company experiment. As shown in "Fig. 4and Fig5".

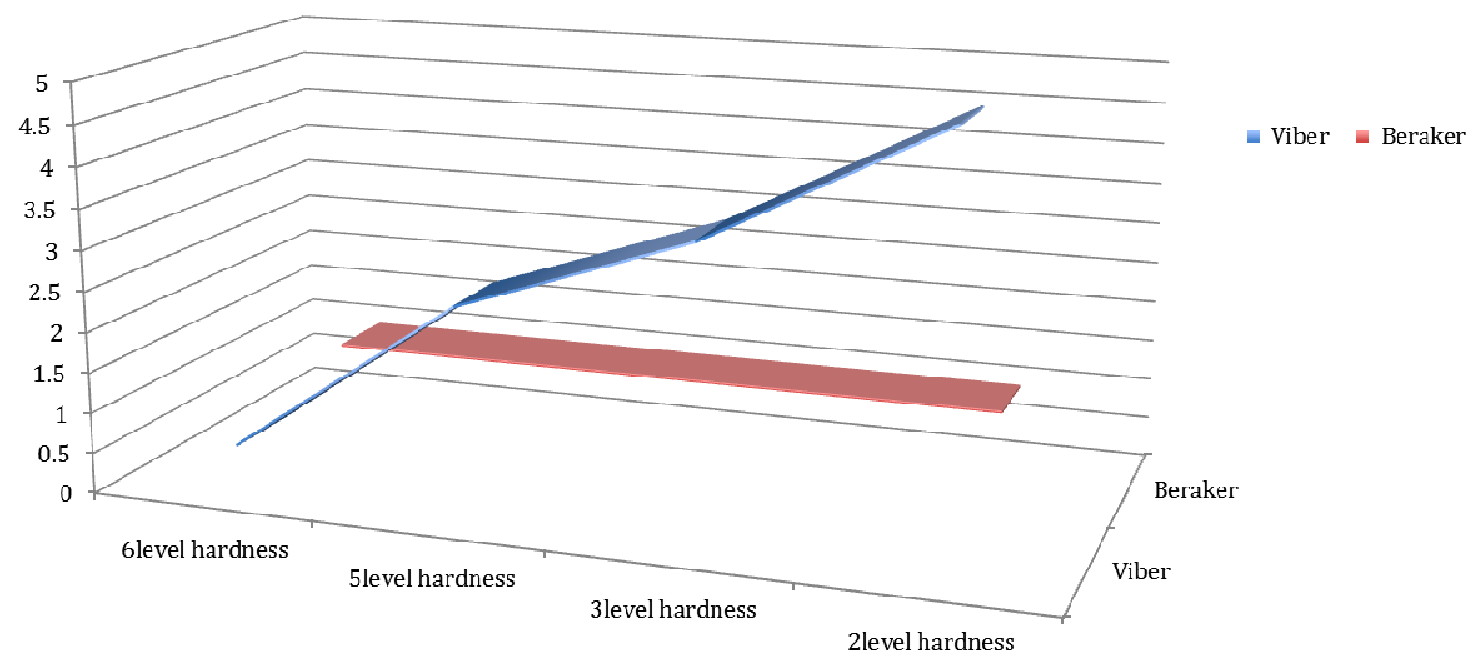

Figure 4. Viber compares with breaker

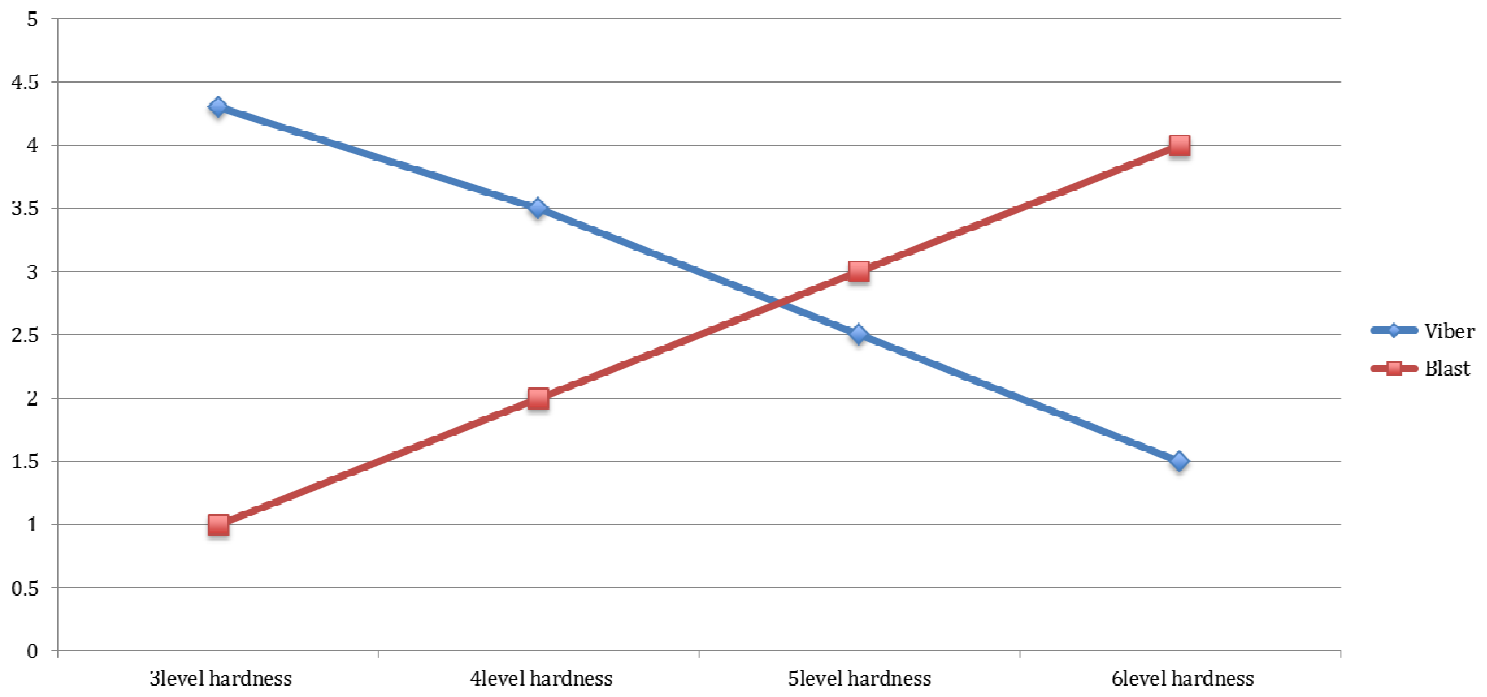

Figure 5. Viber compares with blast

As can be seen form the "Fig.4 and Fig.5", the vibro ripper has a lot of advantages compared with the tradition piston breaker and blast. In practical work, when used in the large-scale earthwork operations, the vibro ripper produced by Shanghai Shing Machinery Co. Ltd, had achieved150 cubic meters by each hour, this result can completely replace the tradition piston breaker. Once the daily output of 3800 cubic meters (22 hours) used in mining, the result completely replace the blasting.

\section{The Key Technical Problems and the Development Trend of Vibro Ripper}

\subsection{The Key Technical Problems}

1) Reconstruction of the vibro ripper main technology, improves the efficiency of crushing ${ }^{[4]}$. 
2) Structural strength of the shell is not enough. The shell have been damaged and torn.

3) Joint bearing wear serious.

4) The bucket teeth material and welding crack. Bucket teeth as the vulnerability accessories, undertakes the important task of breaking in the crushing work and mostly used in the mechanical shovel. So far, there is not a specialized bucket tooth for the vibro ripper.

\subsection{The Development Trendency of Vibro Ripper}

1) The diversification of excitation mechanism. Along with the increasing demand of cracking capacity, in order to improve the cracking efficiency and capacity, the vibration exciter should produce the vibration force as large as possible. This is a key measure of the vibro ripper.

2) Higher utilization of energy ${ }^{[5]}$.

3) The technology of intelligent cracking. According to the size and structural of the broken objects, it could discriminate the broken objects and adjust the rotate speed of hydraulic motor, so as to prolong the vibro ripper working life. We belive that the structures are diversified, the intelligentization and automatization of vibro ripper will appear in the future, accompany with the transformation, the higher stability, quality and efficiency of the vibro ripper will appear.

\section{Summary}

There are several specialties of the vibro, such as high efficient, energy conservation, environmental protection and son on. Predictably, the piston breaker will be taken place by the vibro ripper. China is one of the best manufactures around the world, there are still many problems in the technology aspect at present, we expect to increase R \& D ability and continuous innovation to occupy the domestic market to overseas market.

\section{References}

[1] Si Guimao and Li Xiao ning, development situation and research of hydraulic breaking hammer[J], in:Equipment Management \& Maintenance Technology. 10. (2008).

[2] Cao Yu, Fan Yuxin, a high frequency of broken hammer, $\mathrm{CN}$ 202590877U[P]. China, 12, 12. (2012).

[3] Information on http://vibroripper. com/

[4] Zhou Zhihong, Gao Liwen, Xu Tong-le, analysis of the development and current situation of China's hammer broken hydraulic[J], in:Construction Machinery And Equipment. 8, 35. (2004).

[5] $\mathrm{Xu}$ Tongle, Xia Mingtang, the development and research status of hydraulic breaking hammer[J], in:Hydraulics \& Pneumatics \& Sealing. 6. (2005).

[6] Zhou Jianxin, Zou Xiangfu, Chen Jiangen and He Qinghua, domestic and foreign hydraulic breaking hammer status and development tendency of research and development[J], in:ZaoYan JiXie QiDong GongJu. 4. (2001).

[7] Zhou Zhihong, Ma Fei, the hydraulic hammer of the progress and shortcomings in china[J], in:Construction Machinery And Equipment. 41, 1. (2010).

[8] Gao Junwen, Gao Junfang, high frequency vibration hammer crusher. CN 103233490A[P]. China, 7, 8. (2013).

[9] Cao Yu, Fan Yuxin, Liujian, a high frequency of broken hammer, CN 202055279U[P]. China, 30, 11. (2011).

[10] Information on http://www. fy-power. com/

[11] Information on http://www. gzljyyjx. com/ 